\title{
Development of a Direct Observation Method of Biological Materials for E-TEM
}

\author{
Hiroki Minoda $^{*}$, Tatsuhiro Okabe ${ }^{*}$ and Yuhri Inayoshi ${ }^{*}$
}

* Department of Applied Physics, Tokyo University of Agriculture and Technology, 2-24-16 Nakacho, Koganei, Tokyo 184-8588, Japan,

Structural investigation of protein motion is one of the important ways to understand cell motility. We have studied structural changes of myosin molecules induced by ATP application by using an environmental TEM (E-TEM) $[1,2]$. An image contrast of the myosin heads is very weak due to a weak interaction between myosin molecules and electron beam. Colloidal gold particles were attached to the myosin heads with cite directed antibody, and the structural changes were investigated through the changes in the position of the gold particles. Direct observations of the biological molecules should be the most direct way to study the structural investigations. We have been developing a combination method of E-TEM and a phase contrast TEM (P-TEM) to obtain phase contrast of the biological molecules in water and the biological molecules in water could be visualized [3]. Image contrast was reduced due to increase in background intensity cause by inelastic scattering by ambient water. Intensity of incident electron beam should be suppressed to avoid electron damage of the specimen. Thus, very high contrast imaging is required for direct observation without stain.

We have tried to enhance image contrast by using an objective lens with long focal length (OL-LFL) [4]. A phase plate was set on the selected area (SA) aperture plane and a diffraction pattern was formed by using the OL-LFL on the plane. Figure 1 shows ray diagrams for two optical conditions and figs. 2(a)-(c) are images taken under a conventional TEM condition, a conventional P-TEM condition and the P-TEM condition using OL-LFL. However, black dots correspond to colloidal gold particles are seen in all the images, myosin filaments are seen only by $\mathrm{P}-\mathrm{TEM}$ and there was no contrast in C-TEM. Moreover, the image contrast of the myosin filaments in (c) is higher than that in (b). Thus, it can be claimed that application of the OL-LFL to the P-TEM provided higher phase contrast of phase objects than that obtained by application of a conventional objective lens to the P-TEM. A reason for the contrast enhancement is that high angle scattering waves which give the background intensity were not used for image formation under this optical condition.

\section{References}

[1] H. Sugi, et al. PNAS 105 (2008) 17396-17401

[2] H. Minoda, et al. Biochem. Biophys. Res. Commun. 405 (2011)

[3] Y. Inayoshi, H. Minoda, Y. Arai and K. Nagayama, submitted to Ultramicroscopy.

[4] Hiroki Minoda, Tatsuhiro Okabe, Hirofumi Iijima, in preparation.

[5] This research was partly supported by the Ministry of Education, Science, Sports and Culture in Japan (No. 22540418). 


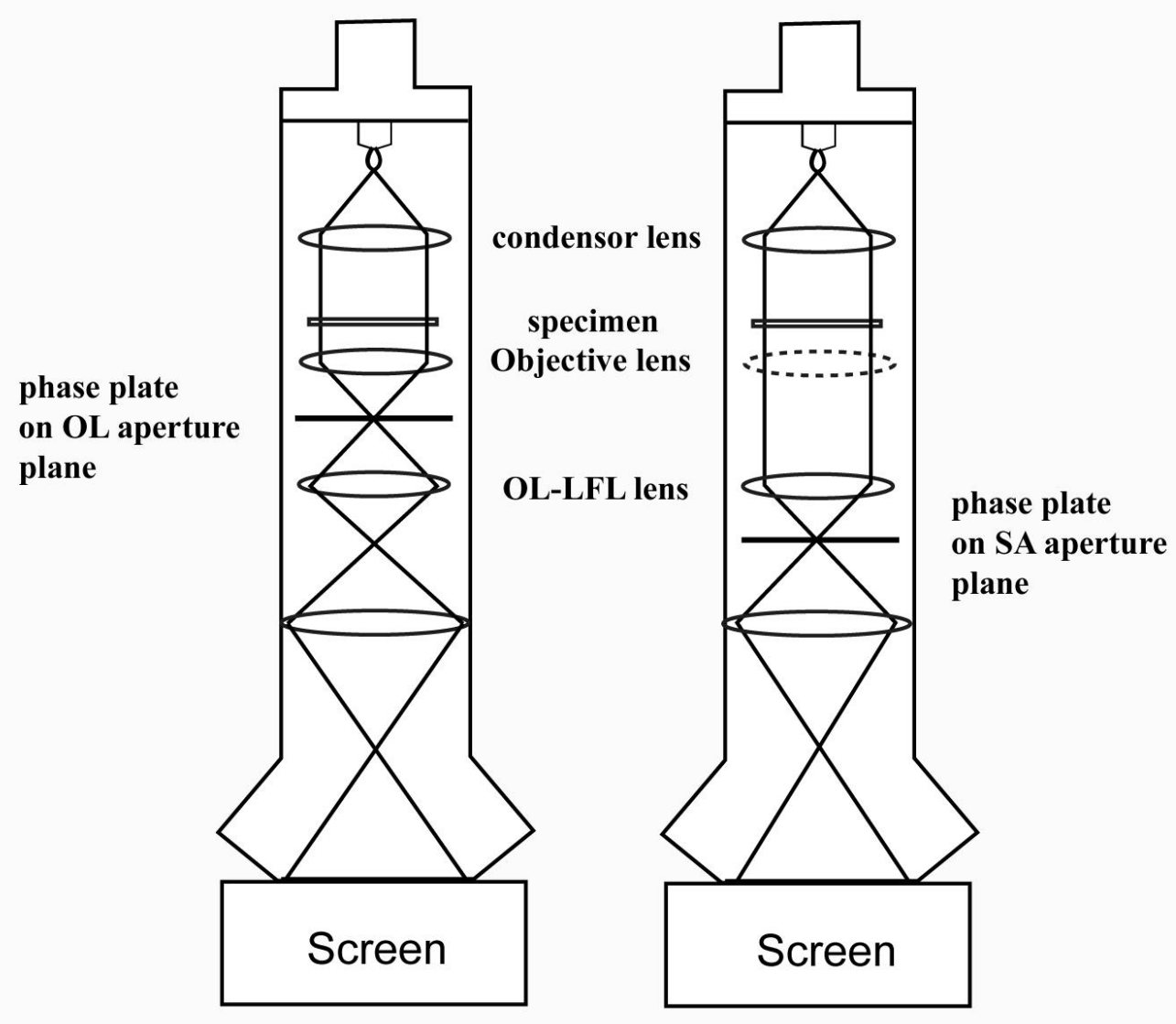

FIG. 1 Ray diagrams showing optical conditions of (a) P-TEM with a conventional objective lens and (b) with the OL-LFL.
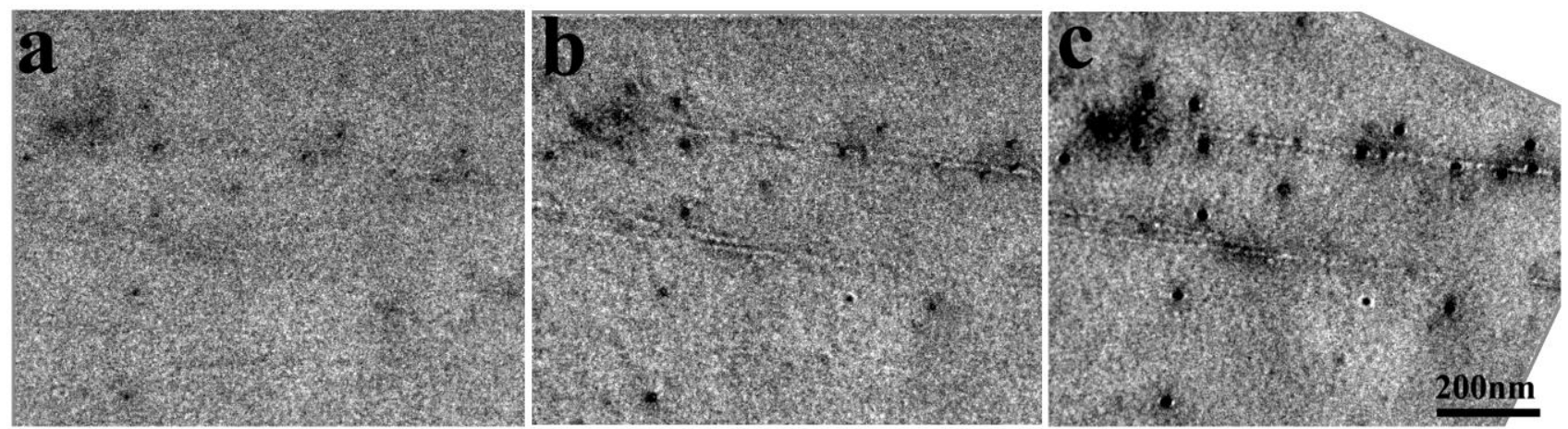

FIG.2. A Comparison of an image contrast of the same area. (a) a conventional TEM image (b) a phase contrast TEM image and (c) a phase contrast TEM image taken by using an objective lens with long focal length. Myosin filaments which can not be seen in (a) can be seen in (b) and (c). This indicates contrast enhancement by P-TEM. The contrast in (c) is higher than that in (b). This is due to the long focal length effect. 